\title{
La musique au service de l'éveil de la nation irlandaise : The Spirit of the Nation
}

Pauline Collombier-Lakeman

\section{(2) OpenEdition}

1 Journals

Édition électronique

URL : http://journals.openedition.org/rbnu/681

DOI : $10.4000 /$ rbnu.681

ISSN : 2679-6104

\section{Éditeur}

Bibliothèque nationale et universitaire de Strasbourg

\section{Édition imprimée}

Date de publication : 1 novembre 2017

Pagination : 60-63

ISBN : 9782859230678

ISSN : 2109-2761

\section{Référence électronique}

Pauline Collombier-Lakeman, « La musique au service de l'éveil de la nation irlandaise : The Spirit of the Nation », La Revue de la BNU [En ligne], 16 | 2017, mis en ligne le 01 juillet 2019, consulté le 10 décembre 2020. URL : http://journals.openedition.org/rbnu/681; DOI : https://doi.org/10.4000/rbnu. 681

\section{(c) (ஒ)(}

La Revue de la BNU est mise à disposition selon les termes de la Licence Creative Commons Attribution - Pas d'Utilisation Commerciale - Partage dans les Mêmes Conditions 4.0 International. 


\section{ORANGE AND GREEN WILL CARRY THE DAP.}

AIR.- "The Protestant Boys."
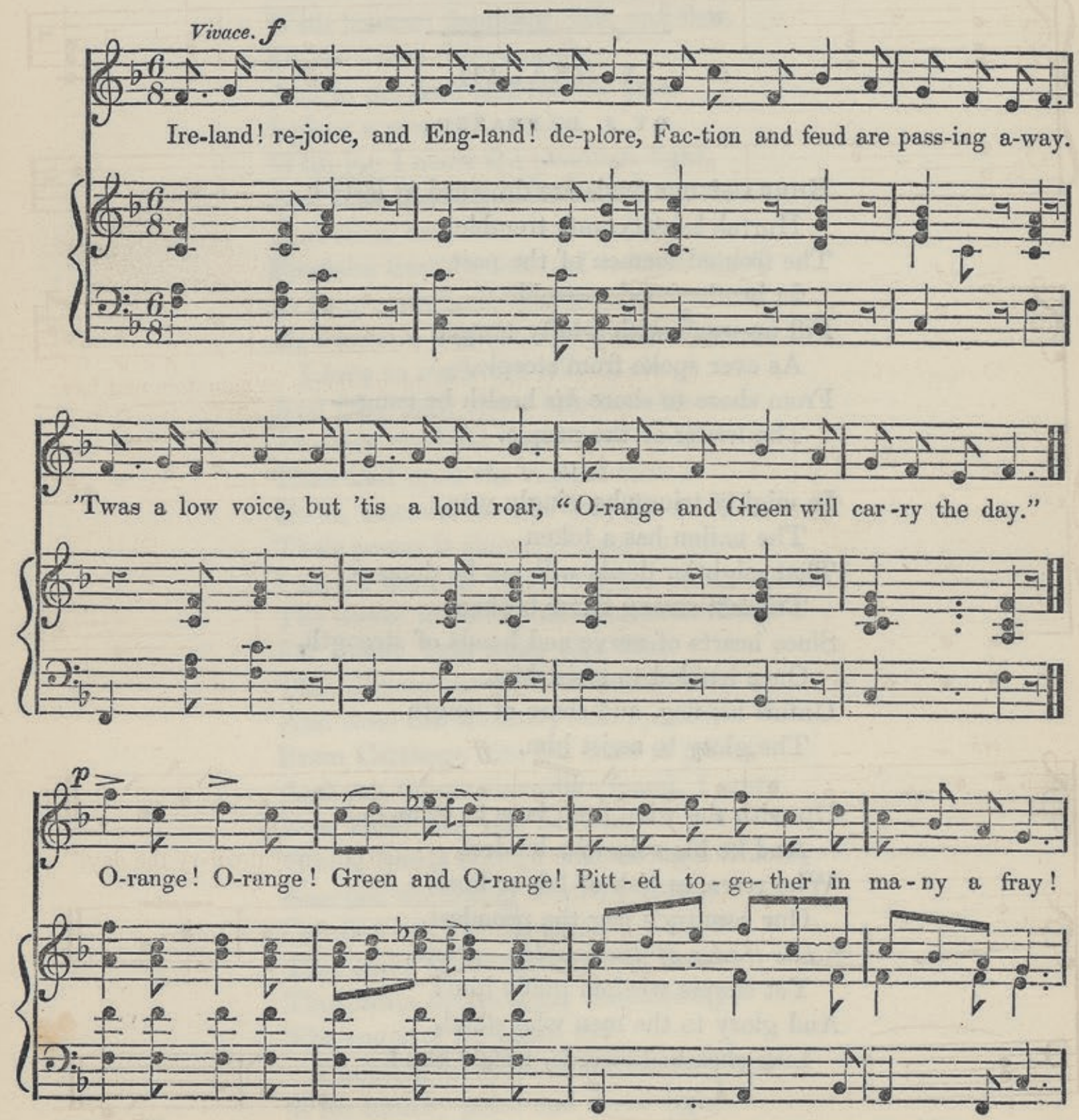

Thomas Davis, " Orange and Green Will Carry the Day » (14 décembre 1844), in The Spirit of the Nation. Ballads and songs by the writers of the "Nation », with original and ancient music, arranged for the voice and piano-forte

[1845], Dublin, James Duffy, 1858 


\section{IRLANDE \\ LA MUSIQUE AU SERVICE DE L'ÉVEIL DE LA NATION IRLANDAISE : THE SPIRIT OF THE NATION

\author{
PAR PAULINE COLLOMBIER-LAKEMAN
}

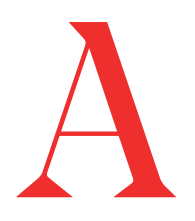

u milieu du $19^{\text {e }}$ siècle, la carte de l'Europe fut considérablement redessinée en raison de l'importance accrue donnée au principe des nationalités. Dans le processus de création de nouvelles identités nationales, la musique sous toutes ses formes (chants populaires ou opéras par exemple) joua un rôle très important. Bien que située à la périphérie de l'Europe, l'Irlande fut exposée aux mouvements politiques, culturels et idéologiques qui se manifestèrent à l'époque. C'est dans ce contexte mouvementé que fut publié, pour la première fois en 1843, le recueil de chants patriotiques The Spirit of the Nation qui a été choisi pour ce numéro de $L a$ Revue de la BNU. Quelques années plus tard, le 7 mars 1848 , un étendard tricolore à trois bandes verticales (l'une verte, la suivante blanche et la dernière orange) fut hissé pour la première fois en public au Wolfe Tone Club de la ville de Waterford par le nationaliste irlandais Thomas Francis Meagher (1823-1867), afin de célébrer la révolution française de février 1848. Meagher s'apprêtait alors à accompagner une délégation de nationalistes irlandais venus apporter un message d'amitié à l'adresse du gouvernement de la République nouvellement établie en France. Lors de son séjour, cette délégation reçut en cadeau un nouvel exemplaire de ce drapeau tricolore irlandais, taillé dans de la soie. La filiation de ce drapeau avec l'étendard tricolore français était évidente.

Ce drapeau n'était pas le seul à l'époque à être utilisé par les nationalistes irlandais. Il n'acquit de statut véritablement officiel qu'en 1937, lorsque son existence fut reconnue dans la constitution qui transforma l'État libre établi en 1922 en une république. Jusqu'au début du $20^{\mathrm{e}}$ siècle, le drapeau tricolore irlandais coexista avec d'autres étendards, dont un de couleur verte décoré d'une harpe, pour représenter l'Irlande aux yeux des nationalistes irlandais.

Que signifiaient les trois couleurs choisies pour l'Irlande ? Meagher lui-même donna la réponse à cette question lorsque le drapeau reçu en cadeau en France fut présenté à un auditoire irlandais à Dublin le 15 avril 1848, peu après le retour de la délégation. Il expliqua que le blanc symbolisait " une trêve durable entre l'orange' et le 'vert' " et affirma espérer que «sous les plis [de cet étendard], les mains des Irlandais protestants et des Irlandais catholiques puissent être 
unies dans une fraternité généreuse et héroïque " (notre traduction). Les premiers mots de cette citation sont encore ceux utilisés aujourd'hui par les services du premier ministre irlandais (taoiseach ${ }^{1}$ ) : ils expliquent les origines et la signification du drapeau national en rappelant que le vert représente l'ancienne Irlande gaélique, tandis que l'orange rappelle le soutien apporté par certains Irlandais au roi Guillaume III (Guillaume d'Orange), au début de la guerre de Neuf $\mathrm{Ans}^{2}$.

Meagher ne fut pas le premier à faire référence à ces trois couleurs comme symboles de l'Irlande et comme emblèmes d'un pays uni et réconcilié. Le 14 décembre 1844, un autre nationaliste, Thomas Davis, avait publié dans The Nation, un périodique à la création duquel il avait œuvré, les paroles d'un chant intitulé «Orange and Green Will Carry the Day ». À lire ce texte dont le titre prédit la victoire des Irlandais protestants et catholiques contre l'occupant anglais, on comprend que Davis, qui était protestant, était lui aussi partisan d'un nationalisme permettant la réconciliation de l'ensemble des Irlandais, toutes obédiences religieuses confondues, et la création d'une nation irlandaise non sectaire.

Ce type de discours est emblématique du mouvement nationaliste dont Davis fut le chef de file, la Jeune Irlande (Young Ireland). On le retrouve aussi fréquemment dans les pages du périodique dont ce mouvement se servit pour promouvoir ses idées, The Nation. Lancé le 15 octobre 1842 par Davis avec l'aide d'un journaliste catholique originaire du comté de Monaghan, Charles Gavan Duffy, et d'un avocat catholique originaire du comté de Mayo, John Blake Dillon, cet hebdomadaire avait pour but de promouvoir non seulement la cause de l'indépendance politique de l'Irlande, mais aussi la culture et l'histoire spécifiques $\mathrm{du}$ pays. The Nation connut un succès immédiat qui ne faiblit pas : le premier numéro se vendit à 12000 exemplaires et le journal devint ensuite le plus lu d'Irlande, avec un lectorat estimé à environ un million de lecteurs.

Une des rubriques les plus populaires dans The Nation était celle intitulée « Poet's Corner», consacrée aux ballades, chansons et poésies. Pour les fondateurs de l'hebdomadaire, les ballades et chansons populaires, si prisées en Irlande, avaient un rôle de premier plan à jouer dans la construction de la nation et de l'identité nationale. Thomas Davis, qui était amateur de musique irlandaise, écrivit ainsi que «le fait qu'un pays fût dépourvu de poésie nationale prouvait sa torpeur désespérante et son absolu provincialisme ".

Les textes publiés par The Nation suscitèrent un tel engouement que, dès janvier 1843, il fut rapporté qu'« au moins vingt textes " de lecteurs avaient été reçus " chaque semaine ". Cet enthousiasme et la conviction que la chanson avait toute sa place dans la lutte nationale poussèrent les rédacteurs du journal à prendre la décision en mars 1843 de publier un recueil des «meilleurs chansons politiques, ballades populaires, épigrammes et pamphlets écrits pour le journal ». Paru deux mois plus tard et vendu à un prix très bas, un recueil de 55 textes (dont 29 associés à des chants populaires), intitulé The Spirit of the Nation, connut un succès retentissant et de multiples rééditions. Une deuxième édition révisée parut ainsi dès septembre 1843. Deux mois plus tard, un deuxième recueil de 50 poèmes (dont 33 inspirés d'airs traditionnels irlandais) vint compléter le premier volume. Ces premières éditions ne contenaient aucune partition de musique, contrairement à celle qui regroupa les deux volumes de textes, accompagnés d'airs anciens mais aussi de compositions inédites. Cette dernière fut publiée sous plusieurs formes afin de séduire des lectorats distincts : d'abord en huit fascicules entre le 29 juin 1844 et le 10 janvier 1845, puis en un ouvrage relié fin janvier 1845. En 1877, la cinquantième édition de ce livre fut publiée. Elle comprenait le texte de Davis « Orange and Green Will Carry the Day ", associé à la transcription d'un air emprunté aux protestants orangistes, « The Protestant Boys ».

Dans la préface à l'édition de 1845 , les auteurs se réjouissent du succès connu par l'ouvrage sous toutes ses formes et décrivent celui-ci comme un phénomène non seulement national, mais mondial : « on peut trouver l'ouvrage partout ", affirment-ils ; « de la cabine de l'amiral de marine anglais à la chaumière du paysan irlandais, de Dublin à Boston, à Sydney et à Calcutta ". L'exemplaire que possède la BNU, et qui date de 1858, atteste effectivement que The Spirit of the Nation connut une diffusion qui dépassa largement les frontières de l'Irlande. Ceci souligne également l'influence de la Jeune Irlande et de son chef de file Thomas Davis, qui fut cruciale à plus d'un 
titre sur l'histoire du pays. En incluant des partitions codifiant un certain nombre de chants traditionnels irlandais et en associant ces airs à des textes, la Jeune Irlande et The Nation contribuèrent à formaliser des mélodies appartenant à la tradition orale et à diffuser plus largement un folklore populaire, à une époque où la langue gaélique était en déclin et où ce déclin fut accéléré par la grande famine de 1845-51. Bien plus, les textes publiés dans The Spirit of the Nation furent érigés en modèles de chants patriotiques et leur succès permit une plus large diffusion des idéaux nationalistes. Davis lui-même servit de source d'inspiration à bien des nationalistes qui lui succédèrent, notamment William O'Brien ou Patrick Pearse, l'un des organisateurs de la rébellion de 1916 à Dublin. C'est d'ailleurs à cette occasion que, le 24 avril 1916, le drapeau tricolore irlandais fut à nouveau hissé au sommet de plusieurs bâtiments occupés par les insurgés, notamment le General Post Office. Après cette date, il fut adopté par ces derniers et, plus largement, par une majorité de la population irlandaise comme le symbole du pays, « le symbole de la seule Patrie, une Patrie indifférente [...] aux mutations successives du pouvoir qui la gouverne et des institutions qui la gèrent ${ }^{3}$.

\section{ORIENTATIONS BIBLIOGRAPHIQUES}

- The Nation (15 octobre 1842-6 janvier 1844 ; 13 juillet 1844-28 juillet 1848)

- Thomas W. Rolleston (éd.), Thomas Davis, Selections from his Prose and Poetry, Dublin, Talbot Press, 1914

- Raoul Girardet, "Les trois couleurs : ni blanc, ni rouge ", in Pierre Nora, Les Lieux de mémoire, tome 1 : La République, Paris, Gallimard, 1984, p. 49-66

- Christine Kinealy, Repeal and Revolution: 1848 in Ireland, Manchester, Manchester University Press, 2009

- Timothy M. Love, « Irish Nationalism, Print Culture and the Spirit of the Nation ", in Nineteenth-Century Music Review (février 2017), p. 1-20

\section{NOTES}

1- On appelle ainsi le premier ministre irlandais (taoiseach signifiant « chef» en gaélique).

2- http://taoiseach.gov.ie

3- Citation empruntée à R. Girardet in Lieux de mémoire, I, 61 (voir Orientations bibliographiques). 